Revista Brasileira de Cartografia

ISSN 1808-0936 | https://doi.org/10.14393/revbrascartogr

Sociedade Brasileira de Cartografia, Geodésia, Fotogrametria e Sensoriamento Remoto

\title{
Níveis de Referência Hidrográfico: uma Abordagem Descritiva
}

\author{
Reference Hydrographics Levels: a Descriptive Approach
}

Raiane Rintielle Vaz Menezes ${ }^{1}$, Larissa Messias de Souza ${ }^{2}$ e Ítalo Oliveira Ferreira ${ }^{3}$

1 Universidade Federal de Viçosa, Departamento de Engenharia Civil, Viçosa, Brasil. rintielle@gmail.com.

ORCID: https://orcid.org/0000-0001-6717-728X

2 Universidade Federal de Viçosa, Departamento de Engenharia Civil, Viçosa, Brasil. larissamessiass@gmail.com.

ORCID: https://orcid.org/0000-0002-7767-1131

3 Universidade Federal de Viçosa, Departamento de Engenharia Civil, Viçosa, Brasil. italo.ferreira@ufv.br.

ORCID: https://orcid.org/0000-0002-4243-8225

Recebido: 08.2020 | Aceito: 03.2021

Resumo: A execução de um Levantamento Hidrográfico (LH) é um processo contínuo, desde o reconhecimento até à aprovação dos resultados nos quais todas as partes envolvidas efetuam um trabalho minucioso. Para tal, é importante ser detentor de conhecimento específico das normas, metodologias e tecnologias utilizadas na hidrografia. Contudo, principalmente no Brasil, o conhecimento, a prática e a literatura acerca de levantamentos hidrográficos ainda atingem menores proporções do que se espera de um país com dimensões continentais e extenso em litoral. A IHO (International Hydrographic Organization) especifica que, para um LH é necessário relacionar todas as profundidades medidas, independentemente do estágio da maré ou do nível da água no momento da sondagem, a um nível de referência hidrográfico comum. Dentro deste contexto, o objetivo deste trabalho é apresentar os principais conceitos relacionados aos níveis de referência considerados nos estudos e levantamentos hidrográficos em áreas costeiras e oceânicas. Realizou-se um levantamento bibliográfico a partir de literaturas, principalmente internacionais, de apoio relacionadas a levantamentos hidrográficos, sua caracterização e metodologias, os quais serviram de apoio aos conceitos aqui apresentados.

Palavras-chave: Níveis de Referência Hidrográfico. Maré. Levantamento Hidrográfico.

\begin{abstract}
The execution of a hydrographic survey is a continuous process, from the recognition to the approval of the results in which all the parties involved carry out a detailed. For this, it is important to have specific knowledge of the standards, methodologies and technologies used in hydrography. However, especially in Brazil, the knowledge, the practice and literature about hydrographic surveys still reach smaller proportions than expected from a country with continental dimensions and extensive in coastline. The International Hydrographic Organization specifies that for an hydrographic survey it is necessary to relate all measured depths, regardless of the tide stage or the water level at the time of the survey, to a common hydrographic reference level. Within this context, the objective of this work is to present on the main concepts related to the reference levels considered in studies and hydrographic surveys in coastal and oceanic areas. A bibliographic survey was carried out based on literature, mainly international, of support related to hydrographic surveys, their characterization and methodologies, which served to support the concepts presented here.
\end{abstract}

Keywords: Hydrographic Reference Levels. Tide. Hydrographic Survey.

\section{INTRODUÇÃO}

Aproximadamente dois terços da superfície terrestre é coberta por água na forma de oceanos, mares, rios, lagoas, reservatórios, entre outros. Muitas atividades de campo são desenvolvidas nesses ambientes e poderão exigir conhecimento prévio das condições do ambiente submerso (GAGG, 2016). Neste sentido, o levantamento hidrográfico, segundo a IHO (2005), contempla informações acerca da batimetria (profundidade), da natureza e geomorfologia do fundo marinho, gravidade, direção e força das correntes oceânicas, altura e fase da maré, magnetismo terrestre, além de determinação das propriedades químicas e físicas da água, com a fundamental finalidade em compilar dados para a geração de cartas náuticas confiáveis. 
De acordo com a Portaria normativa $n^{\circ} 452$ (BRASIL, 2007) os Levantamentos Hidrográficos (LH) realizados em Águas Jurisdicionais Brasileiras (AJB) são especificados pela Marinha do Brasil. A qual é responsável pelo estabelecimento de normas, procedimentos para autorização e controle de levantamentos hidrográficos executados por órgãos ou entidades não pertencentes à Marinha, sendo definidas pela NORMAM-25 (Normas da Autoridade Marítima para Levantamentos Hidrográficos) (DHN, 2017). Essas normas são baseadas nos padrões internacionais ditadas pela Organização Hidrográfica Internacional (IHO International Hydrographic Organization). A NORMAM-25 classifica os levantamentos hidrográficos em duas categorias (ALFA e BRAVO). Os de Categoria A, são levantamentos hidrográficos que devem cumprir integralmente as especificações técnicas, além de serem capazes de fornecer dados que sirvam para a atualização de cartas náuticas ou para as demais finalidades descritas no item 0206 da NORMAM-25. Já a Categoria B, são aqueles levantamentos hidrográficos sem o propósito de atualização de cartas náuticas.

A Marinha do Brasil também é responsável pela produção, atualização e distribuição das Cartas Náuticas em papel, Cartas Raster e Cartas Náuticas Eletrônicas (ENC - Electronic Navigational Chart), por meio da Diretoria de Hidrografia e Navegação (DHN) e do Centro de Hidrografia da Marinha (CHM) (CHM, 2020).

De acordo com a IHO (2005), a carta náutica é um documento cartográfico, decorrente de um LH, proposto para representar a área navegável, fornecendo informações sobre profundidades, natureza do fundo, fundeadouros e áreas de fundeio, perigos à navegação (bancos, pedras submersas, cascos soçobrados, etc.), auxílios à navegação (faróis, faroletes, boias, balizas, luzes de alinhamento, radiofaróis, etc.), altitudes e pontos notáveis aos navegantes, linha de costa e de contorno das ilhas, elementos de marés, correntes e magnetismo. Além dos seus elementos básicos, a carta náutica é um documento de trabalho usado pelo navegador como um mapa da estrada e constitui uma ferramenta de trabalho essencial para a segurança da navegação (MANDARINO; NETO; FOLLY, 2018).

No Brasil, as cartas náuticas não utilizam o mesmo referencial altimétrico das cartas terrestres, isto vem do fato que os dados batimétricos precisam estar relacionados com os efeitos da maré local para permitir uma navegação segura na região. Recomenda-se que o marégrafo esteja referenciado a pelo menos um ponto de referência de nível terrestre. Permitindo-se assim, a integração de referenciais altimétricos terrestres, marítimos e geodésicos nas estações maregráficas (WEELS; KLEUSBERS; VANICEK, 1996; IHO, 2005). Entretanto, alguns países adotam a mesma referência para as cartas náuticas e terrestres, ou seja, uma superfície continua. Por exemplo, no mar Báltico utiliza-se uma referência equipotencial que melhor se ajusta ao Nível Médio dos Mares (NMM). O mesmo ocorre na Holanda, onde os dados de batimetria são referenciados ao quase-geoide (SLOBE, 2013).

O referencial vertical das profundidades representadas em uma carta náutica é denominado Nível de Redução (NR). Consiste num plano de referência que deverá retratar as profundidades mínimas a serem encontradas na região, a fim de eliminar as variações das marés e garantir ao navegante que este encontre raramente profundidade menor do que as representadas na carta náutica (IHO, 2005; FIG, 2006; FRANCO, 2009).

Dentro deste contexto, este artigo foi elaborado por meio de revisão bibliográfica, a fim de unir informações pertinentes sobre os principais níveis de referência hidrográfico aplicados às áreas costeiras e oceânicas, apresentando como estes conceitos e metodologias são importantes para os levantamentos hidrográficos, obras costeiras e outras áreas da ciência. A principal motivação desta revisão se deu para suprir o déficit observado em literaturas brasileiras sobre o assunto, além de incentivar mais pesquisas nesta temática.

\section{DINÂMICA DAS MARÉS}

Segundo IHO (2019), maré é a oscilação periódica vertical da superfície do oceano ou outra grande massa d'água sobre a Terra, resultante da atração gravitacional da Lua e, em menor proporção, do Sol em vários pontos da superfície da Terra.

As forças geradoras de maré são causadas pela combinação das forças gravitacionais e das forças centrífugas entre a Terra, o Sol e principalmente a Lua. A força centrífuga da Terra tem sempre o mesmo 
sentido e magnitude, porém a Lei da Gravitação Universal de Newton (1687) implica que as matérias se atraem diretamente proporcional a suas massas e inversamente proporcional ao quadrado da distância que as separa (HENDERSHOTT e MUNK, 1970). Assim, a força gravitacional entre a Terra e a Lua depende da posição da Lua em relação à Terra, logo a resultante entre ambas as forças gera as marés (QUINN; ATKINSON; WELLS, 2012). Consequentemente, as resultantes dessas forças causam níveis de água maiores, que o normal, nos pontos onde a superfície da Terra está mais próxima da Lua e níveis menores nos pontos mais afastados, representadas respectivamente pelas marés de sizígia e de quadratura (BOON, 2013).

Marés de sizígia ocorrem quando a Lua e o Sol estão em um mesmo alinhamento, produzindo as maiores amplitudes de maré, ou seja, seus efeitos são aditivos. Esta situação ocorre na Lua Nova, quando o Sol e a Lua estão em conjunção, ou Lua Cheia quando estão em oposição. Por sua vez, as marés de quadratura ocorrem nas situações de Lua Crescente e Minguante, quando a posição da Lua e do Sol estão defasadas em um ângulo de aproximadamente $90^{\circ}$, gerando as menores amplitudes de maré. Logo, a variação da maré máxima e mínima ocorrem, aproximadamente, em períodos de 14 dias. (ANTUNES, 2000; WHITE, 2017). A relação das marés com as fases da Lua, representadas pelas posições relativas da Terra, da Lua e do Sol podem ser visualizadas na Figura 1.

Figura 1 - Situações de marés de sizígia e quadratura.

Lua Cheia

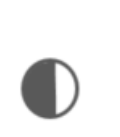

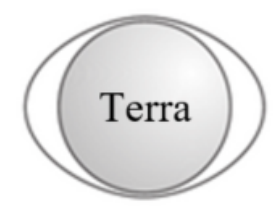

Lua Nova

Sol

Maré de Sizígia

\section{Lua Crescente}
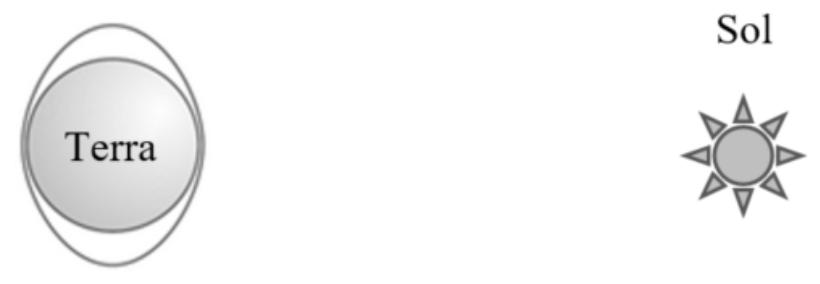

Lua Minguante

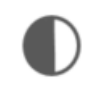

Maré de Quadratura

Fonte: Adaptado de Antunes (2000).

O período das fases da Lua, chamado de mês sinódico ou lunação, é o período médio em que a Lua completa uma revolução em relação à linha que une o Sol e a Terra e é aproximadamente 29,5 dias. Um dia lunar é o tempo que a Terra rotaciona em torno do seu eixo em relação à Lua, resultando em uma revolução a cada 24 horas e 50 minutos. Também é conhecido como dia de maré, por ser a principal causa da ocorrência de variações diárias da maré. Durante um dia lunar, uma Preamar (PM) é identificada quando o nível de água atinge uma elevação máxima e uma Baixa-Mar (BM) quando ocorre um valor mínimo. O intervalo entre sucessivas $\mathrm{PM}$ (e BM) é cerca de 12 horas e 25 minutos (equivalente à metade de um dia médio lunar), logo 
em muitos locais o horário das marés mínimas e máximas ocorrem quase uma hora depois em dias consecutivos (FRANCO, 2009; WHITE, 2017).

Das principais características das marés, a classificação do tipo de maré é fundamental, principalmente, para definir o nível de redução. O tipo de maré refere-se às características entre uma BM e uma PM de um determinado local. Estas características podem ser agrupadas em três classes ou tipos; maré semidiurna, diurna e mista (FRANCO, 2009).

No período de um dia lunar (24h50min) o padrão da maré geralmente é a ocorrência de duas BM e duas PM igualmente espaçadas no tempo. Este padrão é chamado maré semidiurna, sendo pequena a variação entre as alturas das duas BM e duas PM. No entanto, nas marés semidiurnas podem ocorrer elevadas discrepâncias entre as alturas das PM e BM, essas diferenças entre as PM ou BM no mesmo dia lunar são denominadas marés semidiurnas com desigualdades diurnas (LIMA, 2002; FRANCO, 2009).

A maré diurna é caracterizada por apresentar apenas uma BM e uma PM, a cada dia lunar. Nesse tipo de maré, o período da subida e da queda da maré é de aproximadamente 12 horas, em oposição aos períodos de 6 horas das marés semidiurnas. Quando ocorrem duas BM e duas PM com uma grande variação entre as alturas sucessivas das BM ou das PM, considera-se como maré mista. Chama-se de maré mista por possuir características mescladas de marés semidiurna e diurna (IHO, 2005). A Figura 2 ilustra o comportamento da altura em relação ao tempo dos três tipos de marés.

Figura 2 - Comportamento das marés diurna, e mista.
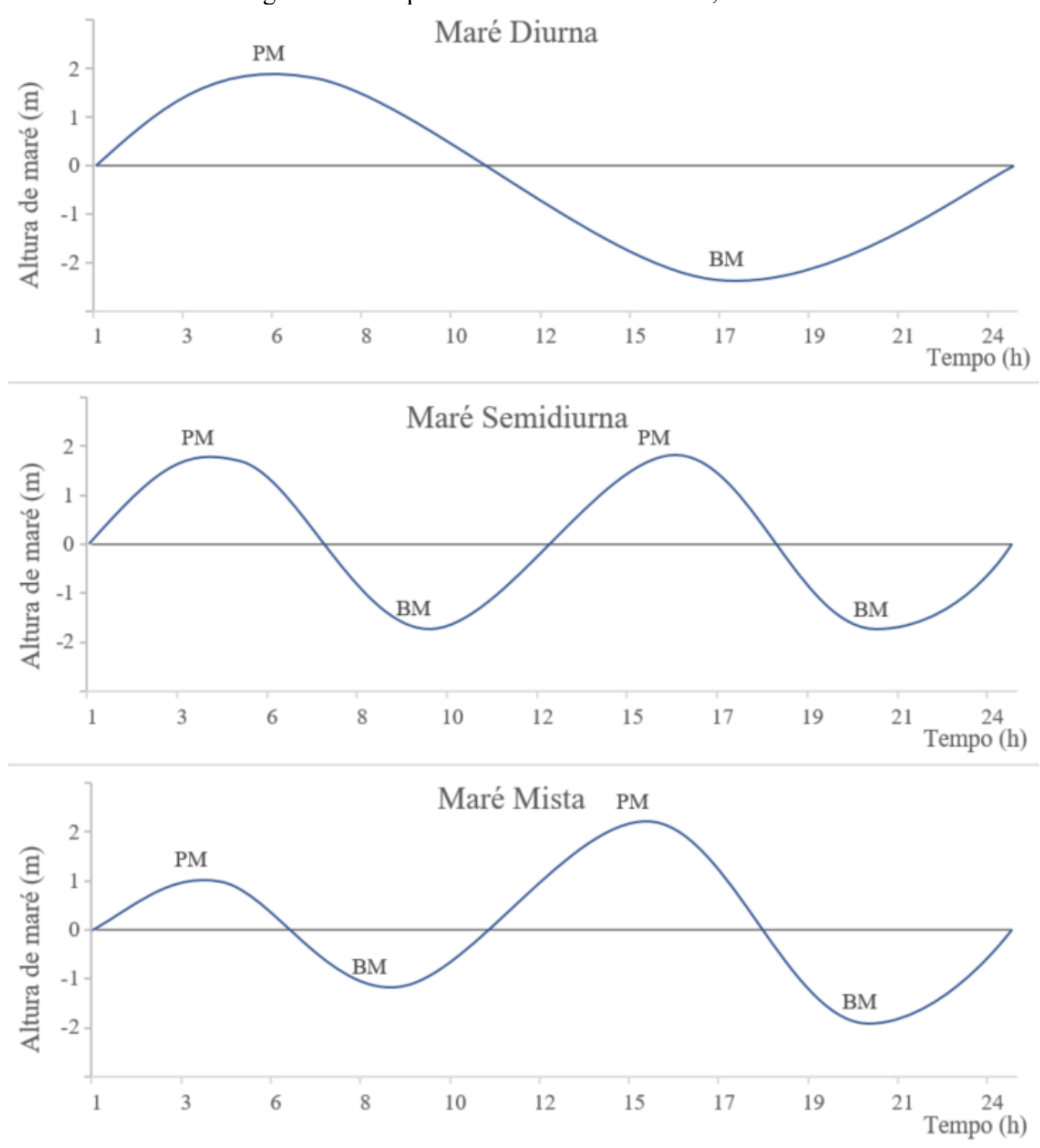

Fonte: Adaptado de IHO (2005). 
A oscilação da maré muda de local para local devido aos efeitos dos continentes, das ilhas, da topografia do fundo, da variação nas órbitas da Lua e do Sol em relação ao Equador e dos efeitos meteorológicos. De acordo com Neto, Saavedra e Ribeiro (2015), de uma forma geral as marés no Brasil têm comportamento semidiurno desde Vitória, no Espírito Santo, até o litoral Norte do país. A região Sul apresenta o mesmo comportamento, no entanto sofre com influencias de maré de origem não-astronômica (MELO FILHO, 2007).

Outro ciclo lunar relevante, é a precessão da órbita lunar ou ciclo nodal lunar. Em que a inclinação da órbita da Lua em relação ao plano equatorial varia de $18,4^{\circ}$ a $28,6^{\circ}$ em um período de 18,61 anos, ou seja, o polo da órbita lunar executa uma revolução completa em torno do polo da eclíptica neste período (o plano da órbita da Lua fica na mesma posição em relação a linha do Equador a cada 18,61 anos). Dessa forma, os padrões de maré se repetem a cada ciclo nodal lunar (PUGH, 1987). Apesar deste padrão resultar em lentas variações ao longo do tempo, é importante considerá-lo em certas determinações de dados maregráficos, principalmente nas previsões de marés.

As previsões de maré podem ser obtidas a partir do resultado da análise harmônica de observações maregráficas. A análise das marés, tem como objetivo reproduzir parâmetros significativos, estáveis no tempo, que descrevem o regime das marés em relação a interação entre a Terra, Sol e Lua no local da observação. Os parâmetros são frequentemente denominados componentes harmônicos, ou de maré, e estão relacionados fisicamente ao processo de geração das marés, além de considerar uma estabilidade de característica regional. Desta forma, pressupõe-se que a partir de uma série suficientemente longa de leituras maregráficas $(>18,61$ anos), é possível definir um valor verdadeiro para cada constante. Na prática, as medições se estendem por períodos finitos, geralmente um ano, um mês ou até alguns dias, e os resultados da análise harmônica dos dados acabam produzindo componentes aproximadas do valor verdadeiro. Logo, quanto maior o período de dados disponível para análise, melhor será a abordagem desses valores reais (PUGH, 2001).

De acordo com Pugh (2004), a lista dos componentes harmônicos é longa, chegando a centenas de componentes. Contudo, na prática poucos componentes são predominantes e suficientes para o cálculo das previsões. A lista dos principais componentes constituintes é apresentada na Tabela 1 e estão separados conforme seu período de influência: longo período ( $>24 \mathrm{~h}$ solares); diurna ( $\sim 24 \mathrm{~h}$ solares) e semidiurna $(\sim 12 \mathrm{~h}$ solares).

Tabela 1 - Principais componentes constituintes.

\begin{tabular}{c|c|c|c}
\hline Tipo & Componente & Símbolo & Período (h) \\
\hline \multirow{3}{*}{ Semidiurno } & Principal Lunar & M2 & 12,42 \\
& Principal Solar & S2 & 12,00 \\
& Maior elíptico Lunar & N2 & 12,66 \\
& Menor elíptico Lunar & L2 & 11,97 \\
& Luni-Solar semidiurno & K2 & 12,01 \\
\hline \multirow{3}{*}{ Diurno } & Maior elíptico Solar & T2 & 23,93 \\
& Luni-Solar diurno & K1 & 25,82 \\
& Principal Lunar & O1 & 24,07 \\
& Principal Solar & P1 & 26,87 \\
& Maior elíptico Lunar & Q1 & 23,10 \\
& Pequeno elíptico Lunar & J1 & 24,84 \\
\hline \multirow{2}{*}{ Longo Período } & Menor elíptico Lunar & M1 & 327,85 \\
& Lunar semimensal & Mf & 661,31
\end{tabular}

Fonte: Adaptado de Pugh (2004). 
A análise harmônica além de considerar os principais componentes harmônicos requer também o conhecimento da amplitude do potencial astronômico, a velocidade angular e a fase de todas as ondas consideradas na análise (MADAH, 2020). Maiores informações sobre análises harmônicas e previsão de maré podem ser encontradas em Doodson (1921), Godin (1972), Pugh (1987) e Franco (2009).

Além das componentes astronômicas representadas pela influência do Sol e Lua nas marés, é importante ressaltar que os elementos e fenômenos meteorológicos, tais como, a temperatura e a pressão atmosférica, a direção e intensidade dos ventos e a passagem de frentes frias afetam diretamente a dinâmica das ondas. Consequentemente, a mesma também sofre influência da profundidade do leito, principalmente nas águas rasas ou de menor profundidade (LIMA, 2002).

Portanto, nos últimos anos muito têm-se notado os fenômenos de "ressacas" em certas localizações do litoral brasileiro, e as mesmas correspondem a ondas com elevadas alturas e em grandes períodos (com variação de 10 ou mais 54 segundos de tempo). Diante desses possíveis fenômenos que assolam o litoral, também se faz necessário para a hidrografia o estudo do atrito das ondas com o leito e suas consequências (ressonâncias, seiches entre outros), além de pesquisas que conduzem ao estabelecimento do nível médio do mar em qualquer localidade (LIMA, 2002).

\section{NÍVEIS DE REFERÊNCIA HIDROGRÁFICO}

A IHO (2019) especifica que, para um levantamento hidrográfico é necessário relacionar todas as profundidades medidas, independentemente do estágio da maré ou do nível da água no momento da sondagem, a um nível de referência hidrográfico comum ou Datum da Carta Náutica (DCN). No Brasil o Datum da Carta Náutica (DCN) é conhecido como Nível de Redução.

Os Níveis de Referência Hidrográfico (NRH) são materializados a partir de constantes harmônicas definidas através da análise de uma série temporal de observações, coletadas numa estação maregráfica específica na costa. Ressalta-se que os NRH, baseados nos níveis de maré, não são superfícies de referência globalmente uniforme, pois variam de local para local. Desta maneira, são estabelecidos com base em medições locais do nível da água nas diversas fases da maré (FIG, 2006).

Devido principalmente ao avanço tecnológico do GNSS (Global Navigation Satellite Systems), da altimetria e gravimetria por satélites, atualmente é possível referenciar dados batimétricos a superfícies contínuas, como, o elipsoide. Uma superfície de referência consistente e estável, para definir um Datum hidrográfico, possibilita um melhor monitoramento da batimetria, rotas marinhas, além da facilidade na transformação entre Data verticais nacionais (WELLS; KLEUSBERG; VANICEK, 1996). Atualmente existem diversos projetos que tem por finalidade conhecer e definir modelos de separação vertical entre as superfícies de referências hidrográficas, como: o BATHYELLI - França, VORF (Vertical Offshore Reference Frame) - Reino Unido, HyVSEPs (Hydrographic Vertical Separation Surfaces) - Canadá, dentre outros. Para maiores informações acerca destes e demais projetos pode-se consultar em Robin et al. (2016) e Rahman e Din, (2020). A Figura 3 ilustra os diferentes NRH tradicionais. 
Figura 3 - Diferentes níveis de referência para levantamentos hidrográficos.

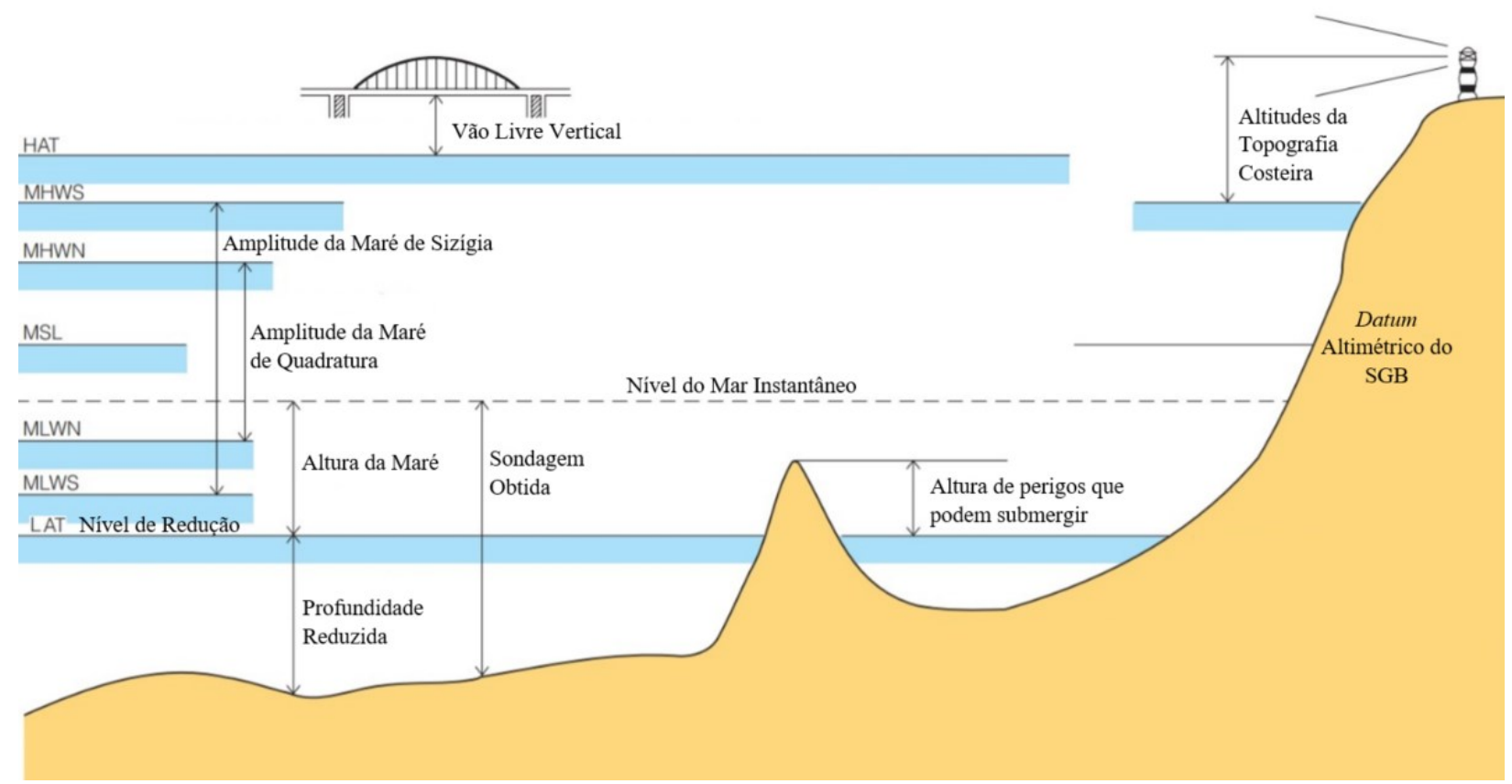

Fonte: Adaptado de IHO (2018).

As marés de Sizígia definem o MHWS (Mean High Water Springs) e o MLWS (Mean Low Water Springs). O MHWS é a média de observações das alturas das preamares de sizígia e o MLWS é a média de observações das alturas das baixa-mares de sizígia. Por sua vez, as marés de Quadratura definem o MHWN (Mean High Water Neaps), que é a média de observações das alturas das preamares de quadratura, e o MLWN (Mean Low Water Neaps) o qual é a média de observações das alturas das baixa-mares de quadratura (WHITE, 2017).

As superfícies definidas pelo HAT (Highest Astronomical Tide) e LAT (Lowest Astronomical Tide) são considerados os níveis preditos extremos, ou seja, são os maiores e menores níveis previstos para ocorrer sob qualquer combinação astronômica e sob condições meteorológicas médias. Ambos são determinados para um local específico e recomenda-se que seja calculado para um período mínimo de 19 anos utilizando as constantes harmônicas derivadas de um período mínimo de 1 ano (IHO, 2005; IHO, 2010).

O MSL (Mean Sea Level) é a média das alturas da superfície do mar em relação a uma superfície terrestre de referência. O nível do mar é registrado por um marégrafo, relacionado a uma régua de marés (IHO, 2018). Este nível abrange diversas proposições e finalidades que serão detalhadas no tópico a seguir.

Destaque deve ser dado que os NRH apresentam variações espaciais e temporais em relação aos Data verticais terrestres, níveis zero, o mesmo ocorre com os Data Verticais Brasileiros (DVB). Isso se dá, devido ao fato de que os NRH são determinações de mínimo, máximo e médio local definidos durante diferentes períodos de tempo. Já o DVB, do Sistema Geodésico Brasileiro (SGB), têm como referência para a componente vertical o NMM originalmente obtido através de observações de séries temporais das alturas das marés tomadas na baía de Imbituba (no litoral de Santa Catarina, entre os anos de 1949 a 1957) e no Porto de Santana (no estado do Amapá, a partir de observações em 1957 e 1958). Logo, os NRH não estão correlacionados aos Data Verticais do SGB (IBGE, 2009). Maiores informações sobre a relação dos DVB e NRH no litoral brasileiro podem ser consultadas em IBGE (2013) e IBGE (2009).

\subsection{Nível Médio do Mar (NMM) e Nível Médio do Mar Local (NML)}

Acreditava-se que o mar oscilava em torno de um nível médio estacionário, isto é, que o nível médio do mar era estático. No entanto, hoje em dia sabe-se que o nível do mar apresenta flutuações em escalas de tempo, estando sujeito a várias forças, como, as astronômicas, ventos, ondas, deflúvios, precipitações, evaporação, temperatura, salinidade, correntes marítimas, geotectonismo, ação antrópica e pressão atmosférica 
(MENEZES, 2007).

MSL é o acrônimo utilizado na literatura inglesa para representar o nível médio do mar, que na literatura brasileira é encontrado, principalmente, como Nível Médio do Mar (NMM) e Nível Médio do Mar Local (NML). Embora sejam diferentes acrônimos, todos expressam a média de leituras do nível do mar tradicionalmente obtidas por marégrafos ou réguas linimétricas, de um determinado local em um período de tempo que registre séries históricas. Além de serem correspondentes, suas respectivas séries temporais são informações relevantes para a definição do Datum Vertical.

Neste sentido, navegantes e hidrógrafos utilizam o termo Nível Médio do Mar Local (NML), não somente por corresponder a um dos níveis de referência hidrográfico, mas por retratar a maré localmente, o que é importante nas áreas costeiras. Como previamente citado, a maré varia de um local a outro e é de extrema valia o conhecimento destas consideráveis oscilações para a segurança da costa e da navegação. Já o NMM é comumente aplicado ao referenciar diretamente a um nível vertical terrestre, ou seja, um Datum geodésico como uma superfície de elevação de nível zero, a qual as alturas dos acidentes topográficos, das curvas de nível, mapas e plantas podem ser referenciadas. No entanto, é importante ressaltar que além dos efeitos da maré, as influências meteorológicas nas estimativas do NMM são expressas por meio do Nível Médio Diário (NMD). Este corresponde a uma escala temporal de $24 \mathrm{hs}$ nas estimativas do nível do mar e para a determinação do mesmo não se deve considerar os fenômenos atmosféricos e/ou meteorológicos de período inferior à escala escolhida (DA SILVA; FREIRE; CRISÓSTOMO, 2014).

A geodesia clássica considerava o NMM, livre de perturbações, como referência teórica que melhor se ajustava ao geoide e assim eram tomados como referenciais verticais terrestres. No entanto, em uma definição atualizada o geoide é considerado uma superfície equipotencial do campo da gravidade global, representado por um valor de referência vertical geopotencial $\left(\mathrm{W}_{0}\right)$. Seu cálculo é fundamentado em modelos de superfícies do mar e no modelo mais recente do campo da gravidade em caráter global. Em vista disso, com o propósito de definir e realizar um sistema único de altitudes, em 2015, a IAG (International Association of Geodesy) convencionou $\mathrm{W}_{0}$ em 62.636.853,4 $\mathrm{m}^{2} \mathrm{~s}^{-2}$, como o valor do potencial do nível de referencial vertical para o Sistema de Referência Internacional de Altitudes (IHRS - International Height Reference System) (IAG, 2015; SÁNCHEZ; SIDERIS, 2017).

Em produtos derivados de altimetria por satélites em conjunto com nivelamentos de alta precisão conectado aos marégrafos, constatou-se diferenças significativas entre os NMM observados ao longo da costa. Tais diferenças podem ter sido influenciadas pelos fenômenos oceânico-atmosférico e os ciclos das chuvas/secas. Outro fator corresponde ao fato dos dados maregráficos necessitarem de correções nos movimentos verticais; ocasionados por marés terrestres, movimentos tectônicos, isostasia, efeitos de carga e movimentos artificiais (induzidos pelo homem), que por sua vez, influenciam diretamente nos registros maregráficos (PUGH, 2004; FRANCO, 2009).

Além desses efeitos, os dados de altimetria por satélites são acrescidos de diversas correções nos efeitos atmosféricos (ionosfera, troposfera seca e úmida) e geofísicos (correções de marés terrestres, oceânicas, polar, efeitos de cargas oceânicas e variações do estado do mar), enquanto os dados de maregrafia são submetidos apenas a eliminação de dados espúrios e outliers. Assim, não havendo suavizações. Desta forma, é possível suavizar os dados de maregrafia a partir da aplicação do filtro de Thompson (1983), onde permite que o usuário suavize as frequências de interesse e otimize seu desempenho por meio da seleção de parâmetros de cálculo; ou o filtro de Godin (1972), que consiste em um filtro de simples implementação, no qual corrige a tendenciosidade dos dados e se baseia na aplicação de três médias móveis (PUGH, 2004; FRANCO, 2009).

Atualmente, tem-se o conhecimento que existe uma separação entre o NMM e o geoide global, denominado de Topografia do Nível Médio do Mar (TNMM, do inglês MDT- Mean Dynamic Topography) (REIS; PALMEIRO; BARBOSA, 2018). A TNMM é uma superfície dinâmica e seu valor médio associado depende do período de avaliação, e pode atingir $\pm 2 \mathrm{~m}$ em relação ao denominado geoide global (FU; CAZENAVE, 2001). Esta variação faz com que os diferentes níveis da maré presentes ao longo da costa do Brasil não representam a mesma superfície equipotencial. Assim, não se pode usar os diferentes NMM ao 
longo da costa como injunção para uma rede altimétrica. Devido a este fato, a maioria da rede vertical brasileira está referenciada ao NMM observada em Imbituba - SC, sendo que a propagação de erros aumenta conforme as Referências de Nível (RRNN) se afastam do Datum (SILVA; FREITAS; DALAZONA, 2016). Mais informações sobre as TNMMs podem ser consultadas nos trabalhos de Seeber (2003), Freitas et al. (2007), Huang (2017), Idžanović et al. (2017), Silva e Guimarães (2018) e Silva e Freitas (2019).

\subsection{Nível de Redução (NR)}

Nos levantamentos hidrográficos, as profundidades que são obtidas incluem a variação do nível das águas, as quais diferem de uma região para outra. Esta variação pode ser por influência das marés ou em decorrência dos períodos de cheias e vazantes de rios. Com o intuito de eliminar esses efeitos, define-se uma superfície de referência em que todas as profundidades sondadas sejam relacionadas a um nível de maré. Segundo a IHO (2005), um plano de referência para a navegação deve ser tão baixo que a maré, em condições normais, não fique abaixo dele. Este plano de referência é denominado Nível de Redução.

As profundidades presentes nas cartas náuticas, estão referenciadas a um NR a fim de suprimir as variações das marés e garantir segurança para que as embarcações, que trafegam naquele local, não encontrem profundidades menores do que as representadas nas cartas. Este mesmo nível também é utilizado na elaboração das tábuas de maré, em que informam a previsão dos níveis de PM e BM de um local diariamente (MIGUENS, 1996).

O NR não é uma superfície de referência uniforme, pois quando estabelecido com base em leituras maregráficas do nível da água, estas variam de local para local. Além disso, as oscilações nos locais dos marégrafos podem não representar a variação das condições hidrodinâmicas que afetam significativamente a amplitude, a fase ou a curva de marés na área da sondagem (muitas vezes, afastadas da costa), ocasionando assim os erros cotidais (FIG, 2014). Estes, são os principais responsáveis por inconsistências em um LH, sobretudo ao uso de uma única estação maregráfica.

Com o intuito de contornar este problema, a DHN sugere realizar um zoneamento geográfico de redução de maré, ou simplesmente, zoneamento de maré. Esta metodologia consiste em interpolar linearmente zonas de marés entre duas ou mais estações maregráficas de forma a evitar inconsistências entre os diferentes níveis de redução na área do LH. Esta metodologia deverá ser aplicada somente onde as influências externas, como, maré, vento, pressão e outras, mantenham suas características constantes nas áreas subdivididas ao longo da área do LH. Maiores informações acerca da metodologia supracitada podem ser consultadas na NORMAM 25 e no site da DHN (DHN, 2017).

Encontra-se na literatura outras metodologias com a finalidade de reduzir os erros cotidais, como, a utilização de modelagem hidrodinâmica no estuário do Amazonas (ARENTZ, 2009), a técnica de interpolação por krigagem bayesiana empírica, nas baías de Buenaventura e Málaga (MACHUCA et al., 2018) e a redução de sondagem a partir da técnica RTK, na baía de Guanabara, descrita por Ramos (2007).

Existem vários planos para determinação do NR, desta forma para as áreas sujeitas a ação de maré oceânica o IHO propõem a utilização do LAT (Lowest Astronomical Tide) ou um nível equivalente próximo, caso seja observado níveis mais baixos do que a LAT, para definição padrão do NR (IHO, 2005).

Entretanto, muitos países utilizam outros níveis de redução. Por exemplo, USA utiliza o MLLW e o Japão o NLLW (Nearly Lowest Low Water) (FIG, 2006). No Brasil, a DHN emprega como NR das cartas náuticas de áreas marítimas da costa brasileira o plano correspondente à aproximadamente à média das baixamares de sizígia (MLWS), pois se utiliza as principais constantes harmônicas, e para os rios adota-se a média das mínimas excepcionais (FERREIRA, 2013).

Soares, Santos e Luz (2018) apresentam o interesse na integração dos sistemas altimétricos e níveis hidrográficos, no Brasil, por meio de um projeto estratégico do IBGE e instituições parceiras. Empenho que se respalda nas recomendações da IHO em ter os NRH conectados ao Datum de referência das altitudes terrestres (IHO, 2008). Maiores detalhes sobre a integração de referenciais altimétricos terrestre e oceânico, pode ser consultado em Santana e Dalazona (2020).

A DHN determina o NR local a partir de observações maregráficas com duração mínima de 32 dias, 
sendo recomendável 1 ano. Estas observações fomentam a identificação das constantes harmônicas, da maré local, a partir da análise harmônica referente ao período temporal observado. Baseado no somatório das amplitudes das principais componentes harmônicas, segundo a classificação das marés pelo critério de Courtier (1938) e Balay (1952), estima-se a semi-amplitude máxima da maré $\left(Z_{0}\right)$. A cota $Z_{0}$ representa o nível que se encontra o NR sob o NML. Ressalta-se, que o NR é definido por critérios puramente astronômicos da maré local e este será sempre referido a um NML. Além disso, tanto o NR quanto o NML devem ser cotados a partir do zero da régua maregráfica (origem das observações maregráficas) (DHN, 2017), conforme ilustra a Figura 4.

Figura 4 - Diagrama dos níveis da estação maregráfica.

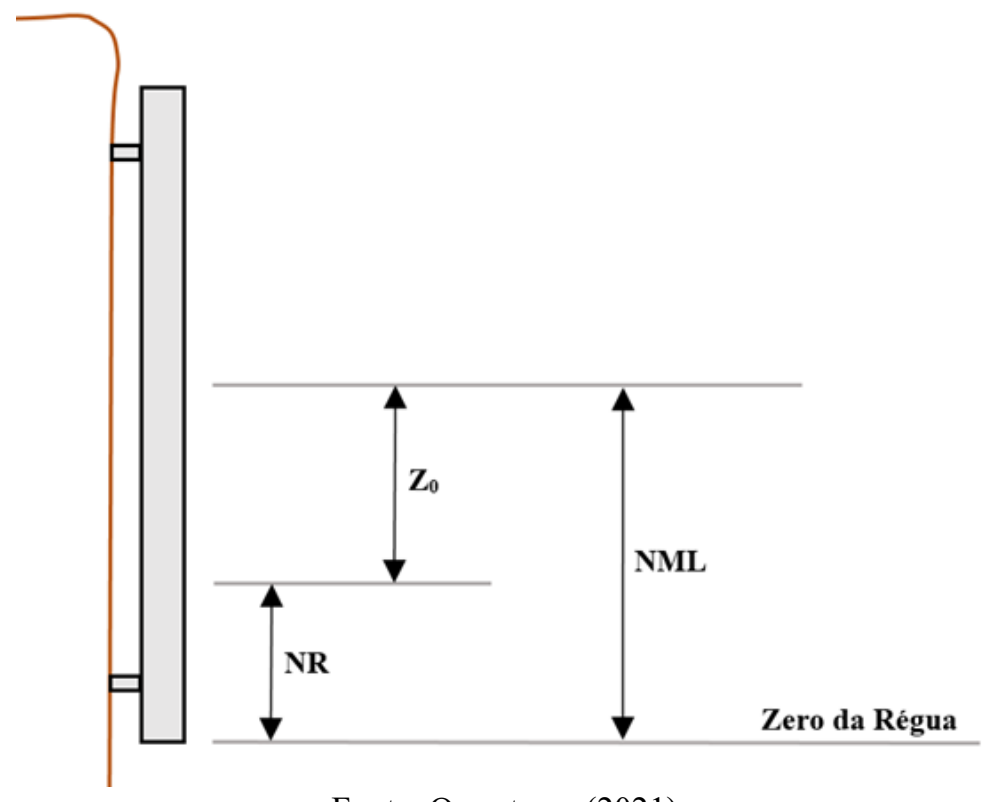

Fonte: Os autores (2021).

Detalhes da metodologia proposta, pela DHN, para o cálculo do NR, bem como para ocupação de novas estações maregráficas e demais cálculos necessários podem sem encontrados na NORMAM 25 e no site da DHN (DHN, 2017).

\subsection{Vão Livre Vertical}

Alturas verticais, consideradas pela navegação marítima, são as medidas de características acima do nível da água. Podem ser divididas em dois grupos gerais: Alturas de objetos que não se tem o interesse em passar por baixo, como, montanhas, faróis, etc. e; Altura dos vãos livres verticais de estruturas nas quais poderá ser necessário passar sob as mesmas, por exemplo, pontes, cabos aéreos energizados e outros.

Vão livre vertical é definido pela IHO (2010) como sendo a altura acima de um nível de referência vertical em que os pontos mais altos de um navio possam passar sob uma estrutura suspensa em segurança, ou seja, é o espaço vertical, sem obstrução física à navegação, entre a parte inferior da estrutura e a maior lâmina d'água prevista para o local (Figura 5). 


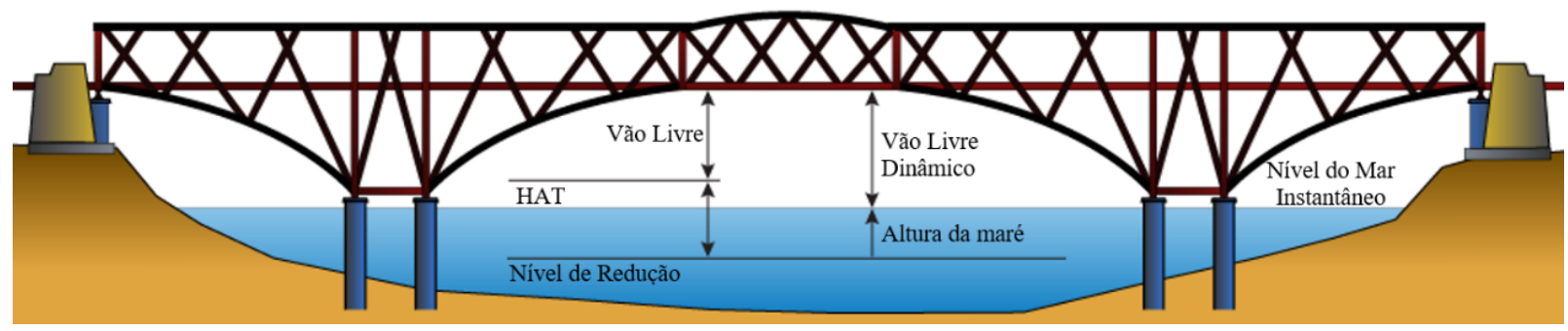

Fonte: Adaptado de SLGO (2020).

O nível de controle vertical para as alturas dos vãos livres, considerado pela IHO, é preferencialmente o plano definido pelo HAT (Highest Astronomical Tide). Pois este, pode ser considerado o nível de maré mais alto previsto para ocorrer sob quaisquer condições meteorológicas médias e astronômicas. Alternativamente pode-se considerar o MHWS como referencial secundário ou outras referências de níveis que melhor representem as preamares específicas do local, estabelecidos por políticas governamentais. Em regiões onde a diferença das marés é insignificante (aproximadamente menor que $30 \mathrm{~cm}$ ) ou nula, pode-se referir à altura dos vãos livres e outras informações de navegação ao NML ou a algum nível aceitável para os serviços hidrográficos (ANTUNES, 2000; IHO, 2018).

A DHN utiliza em suas cartas náuticas a altura dos vãos livres referenciadas ao NML e apresenta em algarismos inteiros o valor referente ao vão livre do obstáculo na própria carta. Se faz necessário verificar a altura da maré no horário e no local da estrutura, a fim de calcular, a partir do NML, se o valor do vão livre obtido naquele horário de maré permitirá uma travessia segura. Alguns navegantes utilizam o termo "calado aéreo" para representar a parte da embarcação que fica fora da água, ou seja, a parte da altura da embarcação que é considerada no cálculo da travessia dos vãos livres.

Em algumas estruturas específicas, como a ponte Presidente Costa e Silva (Rio-Niterói), na qual tem várias alturas de vãos livres a DHN expõe, geralmente atrás da carta náutica, informações detalhadas acerca das alturas dos vãos navegáveis e demais informações e segurança da área. Os navegantes podem consultar o NML e a previsão de maré local no livro Tábua das Marés - Almirante Franco.

\section{CONSIDERAÇÕES FINAIS}

Um levantamento hidrográfico tem-se como finalidade primária a cartografia náutica, porém pode ser realizado para suportar uma variedade de atividades: mapeamento do material do fundo do mar, construção civil, portos e manutenção portuária (dragagem), engenharia costeira a gestão das zonas costeiras, desenvolvimento de recursos marítimos, além de informações sobre reservatórios, barragens e rios.

É de suma importância o conhecimento específico das normas, metodologias e tecnologias utilizadas na hidrografia. No caso do Brasil, o órgão base de controladoria dos levantamentos hidrográficos é a Marinha do Brasil, através da DHN. Há também, uma organização internacional consultiva e técnica de hidrografia, estabelecida por vários países, a IHO.

A Marinha do Brasil em conjunto com a IHO são responsáveis pelo estabelecimento de normas, diretrizes e procedimentos para autorização que norteiam o controle de levantamentos hidrográficos. Neste sentido, a NORMAN-25 (Normas de Autoridade Marítima para Levantamentos Hidrográficos) corresponde a norma, nacional, baseada em padrões internacionais que permitem classificar os levantamentos hidrográficos.

No entanto, a confecção da carta náutica exige o conhecimento dos referenciais de níveis utilizados em um LH e eles podem diferir dos referenciais altimétricos terrestres. Sendo assim, o presente estudo objetivou descrever os principais conceitos acerca dos Níveis de Referência Hidrográfico para estudantes, pesquisadores e profissionais de hidrografia.

Ao focar-se na cartografia náutica e na soberania do país, é possível compreender a importância dos níveis de referência. No Brasil, existem inúmeros portos nos quais a amplitude da maré é tal que estes não 
podem ser demandados na baixa-mar, como é o caso de São Luiz do Maranhão, onde a amplitude de maré alcança incríveis 7 metros. Soma-se a isso, o fato de que as profundidades observadas não podem ser lançadas diretamente na carta náutica, haja vista que cada uma das sondagens corresponde a uma situação momentânea do nível do mar. Assim, no interesse da segurança, as cartas devem indicar as menores profundidades a serem encontradas, daí a importância da definição coerente do nível de redução.

Contudo, a importância do estudo dos níveis de referência vai além daqueles relativos à cartografia náutica, sendo importantes também na geração de energia, biologia (exposição de algas à luz solar), geofísica (determinação do geoide), engenharia costeira (construção de portos, atracadouros, etc.), dentre outros.

\section{Agradecimentos}

Esta pesquisa contou com o apoio da Universidade Federal de Viçosa através do Departamento de Engenharia Civil (DEC), curso de Pós-Graduação em Engenharia Civil, além da Coordenação de Aperfeiçoamento de Pessoal de Nível Superior - Brasil (CAPES) - Código de Financiamento 001, pela bolsa de mestrado concedida ao segundo autor.

\section{Contribuição dos Autores}

Idealização, investigação, revisão da literatura, validação e redação do manuscrito (R. Menezes). Revisão e edição (R. Menezes, L. Souza e Í. Ferreira). Supervisão do projeto (Í. Ferreira).

\section{Conflitos de Interesse}

Os autores declaram que não há conflitos de interesse.

\section{Referências}

ANTUNES, N. S. M. The Importance of the Tidal Datum in the Definition of Maritime Limits and Boundaries. BRADLEY, R.; SCHOFIELD, C. (Ed.). Maritime Briefing, International Boundaries Research Unit: University of Durham, v. 2, n.7, 38 p., 2000. ISBN 1-897643-38-1

ARENTZ, M. F. R. A modelagem hidrodinâmica como auxílio à navegação no canal norte do estuário do Amazonas. 182 f. Dissertação (Mestrado) - Universidade Federal do Rio de Janeiro, Rio de Janeiro, 2009.

BALAY, M. A. La cote du plan. Revue Hydrographic Internationale, v. 24, n. 2, p. 109, 1952.

BOON, John D. Secrets of the tide: tide and tidal current analysis and predictions, storm surges and sea level trends. Elsevier Science, 2013.

BRASIL. Portaria Normativa $n^{\circ}$ 452, 30 de março de 2007. Diário Oficial da União, Brasília, DF, 02 abril. 2007.

CENTRO DE HIDROGRAFIA DA MARINHA (CHM). Cartas Náuticas. Marinha do Brasil, Brasil. 2020. Disponível em: $\quad<$ https://www.marinha.mil.br/chm/chm/dados-do-segnav-cartas-nauticas/cartasnauticas $>$. Acesso em: jan. 2020.

COURTIER, A. Marées. Service Hydrographique de la Marine, p. 234, 1938.

DA SILVA, A. A. A.; FREIRE, E.; CRISÓSTOMO, G. Variações do nível médio anual do mar em Cascais: características e tendências. Estudos do Quaternário/Quaternary Studies, n. 5, p. 51-66, 2014.

DIRETORIA DE HIDROGRAFIA E NAVEGAÇÃO (DHN). NORMAM 25: Normas da Autoridade Marítima para Levantamentos Hidrográficos (2 revisão). Marinha do Brasil, Brasil, p. 94, 2017.

DOODSON, A. T. The harmonic development of the tide-generating potential. Proceedings of the Royal Society of London. Series A, Containing Papers of a Mathematical and Physical Character, v. 100, n. 704, p. 305-329, 1921. 
FERREIRA, Í. O. Coleta, processamento e análise de dados batimétricos visando a representação computacional do relevo submerso utilizando interpoladores determinísticos e probabilísticos. $85 \mathrm{f}$. Dissertação (Mestrado). Universidade Federal de Viçosa, Viçosa, 2013.

FRANCO, A. S. Marés: Fundamentos, Análise e Previsão. Diretoria de Hidrografia e Navegação (DHN), $2^{\circ}$ edição. 344p. Niterói, 2009.

FREITAS, S. R. C.; FERREIRA, V. G.; PALMEIRO, A. S.; DALAZOANA, R.; LUZ, R. T.; FAGGION, P. L. Modelagem do potencial anômalo no Datum Vertical Brasileiro visando a sua nova definição. Boletim de Ciências Geodésicas, Curitiba, v. 13, n. 2, p. 395-419, 2007.

FU, L.; CAZENAVE, A. Satellite Altimetry and Earth Sciences - A handbook of techniques and applications. International Geophysics Series, USA, v. 69, p. 463, 2001.

GAGG, G. Levantamentos Hidrográficos: Noções Gerais. Universidade Federal do Rio Grande do Sul UFRGS, p. 41, 2016.

GODIN, G. The Analysis of Tides. Liverpool University Press, p. 264, 1972.

HENDERSHOTT, M.; MUNK, W. Tides. Annual review of fluid mechanics, v. 2, n. 1, p. 205-224, 1970.

HUANG, J. Determining Coastal Mean Dynamic Topography by Geodetic Methods. Geophysical Research Letters, v. 44, n. 21, p. 11-125, 2017.

IDŽANOVIĆ, M.; OPHAUG, V.; ANDERSEN, O. B. The coastal mean dynamic topography in Norway observed by CryoSat-2 and GOCE. Geophysical Research Letters, v. 44, n. 11, p. 5609-5617, 2017.

INSTITUTO BRASILEIRO DE GEOGRAFIA E ESTATÍSTICA (IBGE). Esclarecimento sobre a relação entre o Datum Vertical do SGB (Imbituba e Santana) e os Níveis de Redução e "Zeros" Hidrográficos no Litoral Brasileiro. 2009. Disponível em: $<$ ftp://geoftp.ibge.gov.br/metodos_e_outros_documentos_de_referencia/outros_documentos_tecnicos/rm pg/relacao_dvsgb_nr_zh.pdf>.

INSTITUTO BRASILEIRO DE GEOGRAFIA E ESTATÍSTICA (IBGE). Relatório de Monitoramento da Variação do Nível Médio do Mar nas Estações da Rede Maregráfica Permanente para Geodésia 2001-2012. Rio de janeiro, $2013 . \quad$ Disponível em:<ftp://geoftp.ibge.gov.br/informacoes_sobre_posicionamento_geodesico/rmpg/relatorio/relatorio_R MPG_2001_2013_GRRV.pdf

INTERNATIONAL ASSOCIATION OF GEODESY (IAG). IAG Resolution (No. 1) for definition and realization of an International Height Reference System (IHRS). Prague, Czech Republic. 2015. Disponível em: $<$ https://iag.dgfi.tum.de/fileadmin/IAG-docs/IAG_Resolutions_2015.pdf $>$. Acesso em: 06 jun. 2020.

INTERNATIONAL FEDERATION OF SURVEYORS (FIG). Publication $\mathbf{n}^{\circ}$ 37: Guide on the Development of a Vertical Reference Surface for Hydrography, p. 30, 2006.

INTERNATIONAL FEDERATION OF SURVEYORS (FIG). Publication $\mathbf{n}^{\circ}$ 62: Ellipsoidally Referenced Surveying for Hydrography, p. 65, 2014.

INTERNATIONAL HYDROGRAPHIC ORGANIZATION (IHO). C-13: Manual on Hydrography. In: International Hydrographic Bureau. Monaco, p. 540, 2005.

INTERNATIONAL HYDROGRAPHIC ORGANIZATION (IHO). M-3: Resolutions of the IHO. Publication n. M-3 - 2 Ed. Monaco: International Hydrographic Bureau, p. 137, 2010.

INTERNATIONAL HYDROGRAPHIC ORGANIZATION (IHO). S-4: Regulations of the IHO for International (Int) Charts and Chart Specifications of the IHO. Special Publication n. $4-4.8$ Ed. Monaco: International Hydrographic Bureau, p. 452, 2018.

INTERNATIONAL HYDROGRAPHIC ORGANIZATION (IHO). S-44: IHO Standards for Hydrographic Surveys. Special Publication n. 44 - 6 Ed. Monaco: International Hydrographic Bureau, p. 47, 2019.

LIMA, O. Localização geodésica da linha da preamar média de 1831-LPM/1831, com vistas à demarcação dos terrenos de marinha e seus acrescidos. 270 f. Tese (Doutorado) - Programa de Pós- 
Graduação de Engenharia Civil, Universidade Federal de Santa Catarina, Florianópolis, 2002.

MACHUCA, M. C. A.; NOSSA, D. A. P.; TRULLO, L. J. S.; BARRERO, F. O. Construcción de la superficie hidrográfica de referencia vertical para las bahías de Buenaventura y Málaga, Pacífico colombiano. Boletín Científico CIOH, v.36, p.53-69, 2018. DOI. 10.26640/22159045.438.

MADAH, F. A. The amplitudes and phases of tidal constituents from Harmonic Analysis at two stations in the Gulf of Aden. Earth Systems and Environment, v. 4, p.321-328, 2020. DOI. 10.1007/s41748-02000152-y

MANDARINO, F.; NETO, A. A.; FOLLY, V. H. M. Inland ENC: Perspectivas para a Cartografia Fluvial no Brasil. Revista Brasileira de Cartografia, v. 70, p. 1551-1565, 2018.

MIGUENS, A P. Navegação: a Ciência e a Arte. Volume I - Navegação costeira, estimada e em águas restritas. Rio de Janeiro: DHN, Brasil, p. 538, 1996.

MELO FILHO, E. Maré Meteorológica na Costa Brasileira. 328 f. Tese Professor Titular - Escola de Engenharia, Universidade Federal de Rio Grande, 2017

MENEZES, D. C. Contribuições metodológicas para a análise de flutuações atmosféricas e oceanográficas de baixa frequência na costa do Estado do Rio de Janeiro. 136f. Dissertação (Mestrado) - Programa de Engenharia Oceânica, Universidade Federal do Rio de Janeiro, Rio de Janeiro, 2007.

NETO, P. B. L.; SAAVEDRA, O. R; RIBEIRO, L. A. S. Optimization of electricity generation of a tidal power plant with reservoir constraints. Renewable Energy, v. 81, p.11-20, 2015. DOI. 10.1016/j.renene.2015.03.011

PUGH, D. T. Tides, Surges and Mean Sea-Level. John Wiley \& Sons. p. 486, 1987.

PUGH, D. T. Tides. In: Encyclopedia of Ocean Sciences. (Ed.). STEELE, J. H.; THORPE, S. A.; TUREKIAN, K. K. Academic Press, San Diego p. 2961-2968, 2001.

PUGH, D. T. Changing Sea Levels: Effects of Tides, Weather and Climate. Cambridge University Press, p. 280, 2004.

QUINN, N.; ATKINSON, P. M.; WELLS, N. C. Modelling of tide and surge elevations in the Solent and surrounding waters: The importance of tide surge interactions. Estuarine, Coastal and Shelf Science, v. 112, p. 162-172, 2012.

RAHMAN, M. F. A.; DIN, A. H. M. The Development of Continuous Hydrographic Datum Using Geodetic Based Approaches: A Review. IOP Conference Series: Earth and Environmental Science, v. 540, 2020.

RAMOS, A. M. Aplicação, investigação e análise da metodologia de reduções batimétricas através do metodo GPS diferencial preciso. 221p. Dissertação (Mestrado). Universidade Federal do Paraná. Curitiba, 2007.

REIS, V. P.; PALMEIRO, A. S.; BARBOSA, L. G. Estudo da obtenção do nível médio do mar com altimetria por satélites. Revista Brasileira de Geomática, v. 6, n.1, p. 3-22, 2018.

ROBIN, C.; NUDDS, S.; MACAULAY, P.; GODIN, A.; BOOM, B. L.; BARTLETT, J. Hydrographic Vertical Separation Surfaces (HyVSEPs) for the Tidal Waters of Canada. Marine Geodesy, v. 39, n. 2, p. 195-222. 2016. DOI: 10.1080/01490419.2016.1160011

SÁNCHEZ, L.; SIDERIS, M. G. Vertical datum unification for the International Height Reference System (IHRS). Geophysical Journal International, v. 209, n. 2, p. 570-586, 2017.

SANTANA, T. A.; DALAZOANA, R. Integração dos Referenciais Verticais Terrestre e Oceânico: Conceitos Relacionados, Projetos Desenvolvidos e Desafios. Revista Brasileira de Cartografia, v. 72, n. 2, p. 345$364,2020$.

SEEBER, G. Satellite Geodesy. $2^{\circ}$ ed. New York: Walter de Gruyter, 2003

SILVA, L. M; FREITAS, S. R. C.; DALAZONA, R. Análise de séries temporais maregráficas correlacionadas 
com observações GNSS no Datum Vertical Brasileiro de Imbituba-SC. Revista Brasileira de Cartografia, v. 68, n. 1, p.73-90, 2016.

SILVA, V. C.; GUIMARÃES, N. Análise do Potencial de Gravidade nas Estações da Rede Maregráfica Permanente para Geodésia Obtido com Diferentes Modelos Geoidais. Anuário do Instituto de Geociências - UFRJ, v. 41, n. 0101-9759, p. 605-613, 2018.

SILVA, L. M.; FREITAS, S. R. C. Análise da Evolução Temporal do Datum Vertical Brasileiro de Imbituba. Revista Cartográfica, n. 98, p. 33-57, 2019.

SLOBBE, D. C.; VERLAAN, M.; KLESS, R.; GERRISTSEN, H. Obtaining instantaneous water levels relative to a geoid with a 2D storm surge model. Continental Shelf Research, v. 52, p. 172-189, 2013.

ST. LAWRENCE GLOBAL OBSERVATORY (SLGO). Dynamic vertical clearance - Summary. 2020. Disponível em: <https://slgo.ca/en/navigation/marine-conditions-clearance/chs-clearance/ summary $>$. Acesso em: abr. 2020.

SOARES, S.; SANTOS, E. G. LUZ, R. T. Rede Geodésica de Referência Costeira (RGRC) como Insumo para Integração de Altitudes e Profundidades: Resultados Preliminares. In: VII Simpósio Brasileiro de Ciências Geodésicas e Tecnologias da Geoinformação, 2018, Recife. Anais... UFPE Programa de Pósgraduação em Ciências Geodésicas e Tecnologias da Geoinformação, p. 742-751, 2018.

WELLS, D. E.; KLEUSBERS, A.; VANICEK, P. A Seamless Vertical-Reference Surface for Acquisition, Management and ECDIS Display of Hydrographic Data. Geodesy and Geomatics Engineering - UNB. Technical Report $\mathbf{n}^{\circ}$ 179. Disponível em: <http://www2.unb.ca/gge/Pubs/TR179.pdf $>$. Acesso em: 10 nov. 2020.

WHITE, J. Tides: The Science and Spirit of the Ocean. Trinity University Press. p. 360, 2017.

\section{Biografia do autor principal}

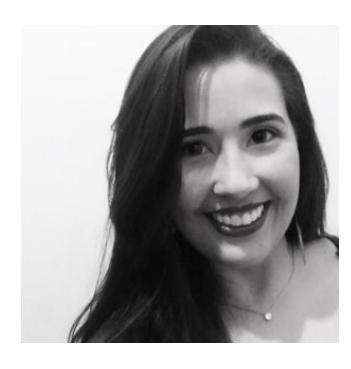

Raiane Rintielle Vaz Menezes, 23 de novembro de 1990. Possui graduação em Engenharia de Agrimensura e Cartográfica pela Universidade Federal de Viçosa (2014). Pós-Graduada em Engenharia de Segurança do Trabalho pela Universidade Cândido Mendes-UCAM (2016). Mestre em Engenharia Civil, área de Concentração em Informações Espaciais (Geodésia) e doutoranda em Engenharia Civil, área de Concentração em Informações Espaciais (Levantamentos Submarinos) pela Universidade Federal de Viçosa. Atua como Engenheira Agrimensora e Cartógrafa na área offshore (surveyor) terceirizada pela Petrobras.

Esta obra está licenciada com uma Licença Creative Commons Atribuição 4.0 Internacional - CC BY. Esta licença permite que outros distribuam, remixem, adaptem e criem a partir do seu trabalho, mesmo para fins comerciais, desde que lhe atribuam o devido crédito pela criação original. 